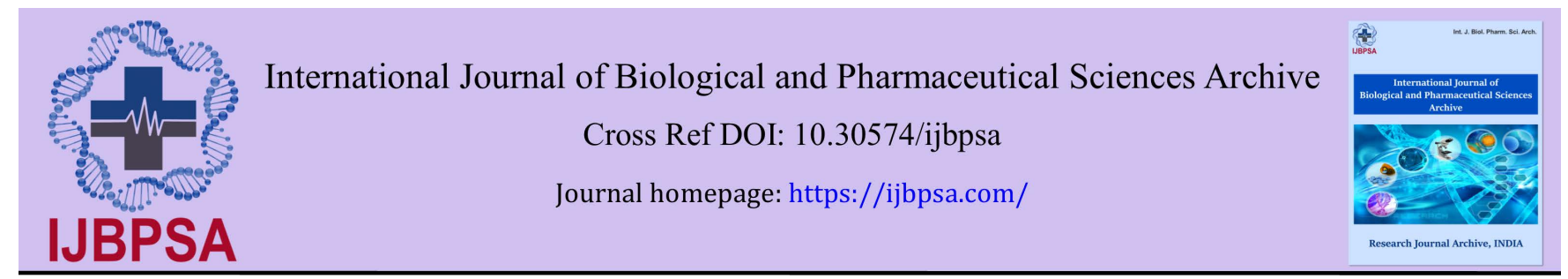

(REVIEW ARTICLE)

\title{
Consequences of COVID-19 on future human genetic composition with special emphasis on homeostasis and natural selection
}

\author{
Rakhad Abdulrazk Alrawi ${ }^{1, *}$, Rafal Abdulrazak Al-Rawi ${ }^{2}$ and Omer Muhi Shareef ${ }^{3}$ \\ ${ }^{1}$ Grand Canyon University and Arizona College. Arizona, USA. \\ 2 Department of Pathology, Hawler Medical University, Erbil, Kurdistan, Iraq. \\ ${ }^{3}$ Al-Rumdi Teaching Hospital for Pediatric and Gynecology, Ministry of Health, Iraq.
}

International Journal of Biological and Pharmaceutical Sciences Archive, 2021, 01(02), 049-056

Publication history: Received on 01 January 2021; revised on 08 January 2021; accepted on 10 January 2021

Article DOI: https://doi.org/10.30574/ijbpsa.2021.1.2.0002

\begin{abstract}
Homeostasis, any self-regulating process by which biological systems tend to maintain stability while adjusting to the condition that is optimal for survival. If homeostasis is effective, life continues; if failed, death succeeds. The stability is a system of dynamic equilibrium, which tends to attain a balance that resists any outside invader that may cause a change. When such a system is disturbed, regulatory genes/immune responds to the departures to establish a new balance; such a process is one of feedback control. There are many kind of homeostasis, among are physiological homeostasis and genetic homeostasis. The first is essential to maintain conditions within restricted limits. Otherwise, the individual will fail to function properly such as body temperature, blood sugar level, blood Pressure and blood pH regulations. On the other hand, genetic homeostasis pertaining to population (may be the whole global population of 7.8 billion) when the gene pool is expressing frequencies of alleles and consequently population genetic frequencies remain the same over a period of time, indicating, the tendency of a population to equilibrate its genetic composition and to resist any changes. In this article it was discussed the consequences of COVID-19, as an evolutionary force of natural selection to the genetic composition of human population. It was concluded that physiological homeostasis are more stable than genetic homeostasis. It was suggested that global population into six genetic categorization for resistant versus susceptible individuals to COVID-19 incidence. Researchers can explore the potential role of genome wide association (GWAS), as molecular genetic markers to be used as early detection of individuals susceptible to COVID-19 to have intensive care.
\end{abstract}

Keywords: COVID-19; Natural selection; Physiological homeostasis; Genetic homeostasis; Genetic composition

\section{Introduction}

Homeostasis refers to the ability of an organism to maintain a state of internal balance and physical wellbeing in case of changes or outside factors. Successful homeostasis is vital to the survival of any living thing, and being able to maintain homeostasis even in adverse conditions is one of the most important evolutionary advantages.In biology, homeostasis is the state of steady internal, physical, and chemical conditions maintained by living systems, within certain pre-set limits (range)[1].

This is the condition of optimal functioning for the organism and includes many variables, such as body temperature, blood sugar level, and these need to be regulated despite changes in the diet, disease incidence, or level of activity. Each of these variables is controlled by one or more regulators or homeostatic mechanisms, which together maintain life.Homeostasis is brought about by a natural resistance to change when already in the optimal conditions [2, 3].

${ }^{*}$ Corresponding author: Rakhad Alrawi

Grand Canyon University and Arizona College. Arizona, USA.

Copyright (C) 2021 Author(s) retain the copyright of this article. This article is published under the terms of the Creative Commons Attribution Liscense 4.0. 


\subsection{Homeostasis}

The ability of a system or living organism to adjust its internal environment to maintain a stable equilibrium, such as the regulation of blood pressure, as well as the ability of homoeothermic animals to maintain a constant body temperature. Two main mechanisms control homeostasis:

- Negative feedback mechanisms reduce output or activity to return an organ or system to its normal range of functioning. Regulation of blood pressure is an example of negative feedback. Blood vessels have sensors called baroreceptors that detect if blood pressure is too high or too low and send a signal to the hypothalamus. The hypothalamus then sends a message to the heart, blood vessels, and kidneys, which act as effectors in blood pressure regulation. If blood pressure is too high, the heart rate decreases as the blood vessels increase in diameter (vasodilation), while the kidneys retain less water. These changes would cause the blood pressure to return to its normal range. The process reverses when blood pressure decreases, causing blood vessels to constrict and the kidney to increase water retention.

- Positive feedback: A feedback loop in which the output of a system is increased by the mechanism's own influence on the system that creates that output. Positive feedback is a mechanism in which an output is enhanced in order to maintain homeostasis. Positive feedback mechanisms are designed to accelerate or enhance the output created by a stimulus that has already been activated. Positive feedback mechanisms are designed to push levels out of normal ranges. A positive feedback example is blood platelet accumulation and aggregation, which in turn causes blood clotting in response to an injury of the blood vessels.

Moreover,homeostasis is maintained by different mechanisms. All homeostatic control mechanisms have at least three interdependent components for the variable being regulated:

- A sensor or receptor detects changes in the internal or external environment. An example is peripheral chemoreceptors, which detect changes in blood $\mathrm{pH}$.

- The integrating center receives information from the sensors and initiates the response to maintain homeostasis. The most important example is the hypothalamus, a region of the brain that controls everything from body temperature to heart rate, and blood pressure.

- An effector is any organ or tissue that receives information from the integrating center and acts to bring about the changes needed to maintain homeostasis. One example is the kidney, which retains water if blood pressure is too low [4].

On the other hand, the partial pressure of oxygen in the blood, the amount of oxygen that can be carried, depends on the hemoglobin content. The partial pressure of oxygen may be sufficient for example in anemia, but the hemoglobin content will be insufficient and subsequently as will be the oxygen content. Given enough supply of iron, vitamin B12 and folic acid, EPO can stimulate RBC production, and hemoglobin and oxygen content restored to normal [ 5, 6].

Many diseases are the result of a homeostatic failure. Almost any homeostatic component can malfunction either as a result of an inherited defect, an inborn error of metabolism, or an acquired disease. Some homeostatic mechanisms have inbuilt redundancies, which ensures that life is not immediately threatened if a component malfunctions; but sometimes a homeostatic malfunction can result in serious disease, which can be fatal if not treated. A well-known example of a homeostatic failure is shown in type 1 diabetes mellitus. Here blood sugar regulation is unable to function because the beta cells of the pancreatic islets are destroyed and cannot produce the necessary insulin. The blood sugar rises in a condition known as hyperglycemia. The plasma ionized calcium homeostat can be disrupted by the constant, unchanging, over-production of parathyroid hormone by a parathyroid adenoma resulting in the typically features of hyperparathyroidism, namely high plasma ionized $\mathrm{Ca}^{2+}$ levels and the resorption of bone, which can lead to spontaneous fractures. The abnormally high plasma ionized calcium concentrations cause conformational changes in many cell-surface proteins (especially ion channels and hormone or neurotransmitter receptors)giving rise to lethargy, muscle weakness, anorexia, constipation and labile emotions [7, 8]. Risk and stresshomeostasis, the tendency of a population or an individual to stay at a certain level of risk and stress [9].

\section{Natural Selection}

It was reported that population is characterized by a dynamic variability of the genetic composition, lending emphasis to the concept of an irregularly shifting state of genetic balance. This may cause integrative properties of populations to assume the importance in evolutionary process dealing with differentiation between populations, as well as genetic phenomena within populations. In large populations, whereby in the absence of selection, mutation or migration they 
tend to maintain their genetic composition constant. Yet it is the genetics behavior of the units of inheritance linking individuals of successive generations[10]. Genetic variation occurs among the individuals of any population of organisms. Some differences may improve an individual's chances of surviving and reproducing, which mean that it leaves more offspring, causing evolutionary change [11]. The concept of fitness is central to natural selection. In broad terms, individuals that are more "fit" have better potential for survival, as in the well-known phrase "survival of the fittest". Consequently, its genes become more common in the population of the next generation [12].

Furthermore, in biology, competition is an interaction between organisms in which the fitness of one is lowered by the presence of another. This may be because both rely on a limited supply of a resource such as food, water, or land. Competition may be within or between species, and may be direct or indirect. Species less suited to compete should in theory either adapt or die, therefore, competition plays a powerful role in natural selection [13, 14]. A prerequisite for natural selection to result in adaptive evolution, novel traits and speciation is the presence of heritable genetic variation that results in fitness differences. Genetic variation is the result of mutations, genetic recombination and alterations in the number, shape, and size of the chromosomes. Any of these changes might have an effect that is highly advantageous or highly disadvantageous. In the past, most changes in the genetic material were considered neutral because they occurred in non-coding DNA or resulted in a synonymous substitution. However, many mutations in non-coding DNA have deleterious effects [15 - 17].

Some mutations occur in "regulatory genes. Changes in these often have large effects on the phenotype of the individual because they regulate the function of many other genes. Most, but not all, mutations in regulatory genes result in nonviable embryos. When mutations result in a higher fitness, natural selection favors these phenotypes and the novel trait spreads in the population. Established traits are not immutable; traits that have high fitness in one environmental context may be much less fit if environmental conditions change.

\section{Genetic composition of biological populations}

Population genetics is a field of biology that studies the genetic composition of biological populations, and the changes in genetic composition that result from the operation of various factors, including natural selection. Population geneticists pursue their goals by developing mathematical models of gene frequency dynamics (change in gene frequency). COVID-19 as an evolutionary force of natural selection is most important factors that can affect a population's genetic composition. Natural selection occurs when some variants in a population out-reproduce other variants as a result of being better adapted to the environment, or 'best fit'. Presuming the fitness differences are at least partly due to genetic differences, this will cause the population's genetic makeup to be altered over time. It was states that if mating is random in the population, and if the evolutionary forces of natural selection, mutation, migration and drift are absent, then in the offspring generation the genotypic and allelic frequencies can be related and then be in stable and constant over next generations [18]. Nevertheless, when mating is not random in small population (random genetic drift, refers to the chance fluctuations in gene frequency that arise in finite populations), as well as natural selection, mutation, migration, and COVID-19 can change the genotype composition of the population. Hence, COVID-19 occurs when some genotypic variants in a population enjoy a survival or reproduction advantage over others [19 -21].

\section{Population differentiation in disease-related genes}

Studies of the pattern of molecular evolution of human disease-related genes will provide insight into the origin, maintenance and mechanism of disease. Previous reports suggested that disease-related genes tend to evolve under purifying selection based on the comparison of non-synonymous rate to synonymous substitution rates [22, 23].

\section{Human variation in gene expression}

Human genetic variation is the genetic differences both within and among populations. There may be multiple variants of any given gene in the human population, leading to polymorphism. Some genes are not polymorphic, meaning that only a single allele is present in the population, the gene is then said to be fixed. No two persons are genetically identical. On average, in terms of DNA sequence, each human is $99.5 \%$ similar to any other human. Even monozygotic twins, who develop from one zygote, have infrequent genetic differences due to mutations occurring during development. Differences between individuals are the key of technique such as genetic fingerprinting. Human genetic variation was noticed among tissues, individuals, strains, and populations. The interactions among these sources of variation are relevant for patho-physiological investigations such as cancer and heart failure to be associated with changes in tissuespecific gene expression. Differences among tissues suggesting that these measures of gene expression accurately reflect changes in proteins and their phenotypic effects [24]. 


\section{Variation among individuals}

Variation in gene expression among healthy male individuals raised under controlled laboratory conditions was high. Nearly half of the metabolic genes (48\%) were differentially expressed among individuals within a population for any one tissue. Differences in gene expression among individuals are unlikely to be due to common reversible environmental factors that affect physiological performance (acclimation effects) [25]. Although inter-individual variance in gene expression seems prevalent, our observation that $48 \%$ of genes are differentially expressed among individuals is high $[26,27]$ A variety of factors can contribute to differences in gene expression among individuals. Pritchard et al. (2001) proposed that differences in immune status may explain the 3.3\% difference in gene expression among genetically identical mice. 28 Sex explained a large portion of among-individual variation in gene expression, whereas genotype was less of an influence, and the influence of age was weak [29].

\section{Variation among tissues}

Another important source of biological variation in gene expression is differences in expression among different tissues; $76 \%$ of genes were differentially expressed between brain, heart and liver, and expression in the liver was the most distinct compared to heart and brain. In this study, genes printed on our array are primarily enzymes functional in central metabolic pathways such as fatty-acid metabolism, glycolysis and oxidative phosphorylation. Of the oxidative phosphorylation genes differentially expressed between tissues, 92\% were more highly expressed in heart or brain than in liver. The primary purpose of the heart is to act as a pump, and contraction is highly dependent on oxidative metabolism [30]. The metabolic rate in the brain is 7.5 times the average rate in the rest of the body [31]. High metabolic demand in the brain supports pumping of ions across neuronal membranes during action potentials and metabolism is primarily oxidative. Mitochondria are the principal sites for oxidative phosphorylation, and are most numerous in heart, brain and skeletal muscle cells. The liver, in contrast, is much more functionally diverse, as it is involved in carbohydrate storage, synthesis of proteins, glucose, fatty acids, cholesterol and lipids, and metabolism of xenobiotics and endogenous compounds, and has a relatively low respiration rate. Accordingly, transcripts of genes functional in oxidative phorphorylation appear to represent a much smaller portion of the cell's RNA transcripts in liver tissues than in the heart or brain. In addition, genes involved in fatty acid and phospholipid synthesis were more highly expressed in liver than the other tissues. Differences in expression among tissues detected using our array appear to reflect differences in the metabolic status of brain, heart, and liver. Because data presented here support well established patterns of metabolism, they suggest that measuring mRNA expression using microarrays accurately reflects changes in proteins and their phenotypic effect [32]. Understanding patterns of gene-expression variation within and among human populations will provide important insights into the molecular basis of phenotypic diversity and the interpretation of patterns of expression variation in disease. However, little is known about how gene-expression variation is apportioned within and among human populations. It was indicated that extensive variation in gene-expression levels and estimate that $83 \%$ of genes are differentially expressed among individuals and that $17 \%$ of genes are differentially expressed among populations. By decomposing total gene-expression variation into within- versus among-population components, we find that most expression variation is due to variation among individuals rather than among populations, which parallels observations of extant patterns of human genetic variation [33].

Study of human genetic variation may be important because some disease-causing alleles occur more often in people from various geographic regions. Differences in allele frequencies contribute to group differences in the incidence of some monogenic diseases, and they may contribute to differences in the incidence of some common diseases. To the extent that ancestry corresponds with racial or ethnic groups or subgroups, the incidence of monogenic diseases can differ between groups categorized by race or ethnicity. With many diseases involving numerous genetic variants and environmental factors, investigators point to evidence suggesting the involvement of differentially distributed alleles with small to moderate effects. However, allelic variation in a susceptibility gene been may account for a significant fraction of the difference in disease prevalence among groups, and the role of genetic factors in generating these differences remains uncertain. The structure of human populations is relevant in various epidemiological contexts. Because of variation in frequencies of both genetic and nongenetic risk factors, rates of disease and of such phenotypes as adverse drug response vary across populations. Further, information about a patient's population of origin might provide health care practitioners with information about risk when direct causes of disease are unknown.

\section{What future developments can be expected?}

A growing number of research groups are exploring the potential role of genome wide association studies (GWAS) may play in susceptibility/resistance of COVID-19. It was reported that not yet clear why some people infected with Severe Acute Respiratory Syndrome Corona virus 2 (SARS-CoV-2), the virus that causes COVID-19, get sick, while others have 
only mild symptoms. On the other hand, some are not infected. There is some evidence that scientists know that people's genes can influence how their bodies react to viruses. Researchers described a genome-wide association study (GWAS) of samples from 1,610 hospitalized patients with COVID-19 and 2,205 healthy controls. It was identified variants in two regions-the locus that encodes blood type and a multi-gene cluster on chromosome 3-that were linked to respiratory failure during SARS-CoV-2 infection. Furthermore, a number of studies have pointed to the potential role that an individual's blood group may have on their susceptibility to COVID-19. The pathogenesis underlying severe COVID-19 and associated symptoms is, unfortunately, yet to be fully understood. Researchers decided to look at the infectious disease through a genomic lens to explore whether variants in the genome could underlie clinical variability in COVID19 symptoms.

The scientists tested for a GWAS methodology, in which common variants in a number of individuals, both without the disease (control groups) and with the disease (COVID-19 patients) are explored using arrays. Through this approach, genetic variants that are associated with the disease will be found at a higher frequency than in the control groups.

Moreover, the scientists discovered two independent gene variants that appear to be associated with COVID-19 induced respiratory failure: rs11385942 at locus 3p21.31 and rs657152 at locus 9q34.2 - these were significant at the genome wide level $(\mathrm{P}<0.001)$. Looking closer at chromosome 3p21.31, the peak association signal encompassed a cluster of six genes: SLC6A20, LZTFL1, CCR9, FYC01, CXCR6, and XCR1. Whilst a causative gene cannot be drawn reliable result. It's important to note that GWAS studies do not demonstrate a causal role for a specific genetic variant - rather, it points to associations [34 - 40].

Therefore, according to the above discussion, COVID-19 can be considered as one of the evolutionary force of natural selection, which can cause differential survival and reproduction of individuals due to genetic differences in diseases incidence. It is a key mechanism of evolution, the change in heritable traits of a population over time. Currently, the outbreak is known to have caused over 24 million cases and taken more than 850000 lives the world over. However, huge gaps remain in the scientific understanding of how the virus causes a spectrum of disease ranging from asymptomatic to lethal respiratory failure. At present, the incubation period of the virus is thought to be around 5 days, and almost $98 \%$ of cases become symptomatic within 11-12 days of infection. The challenge to researchers today is to understand the pathogenesis and mode of transmission of the agent. One significant component of this is to identify the host and agent factors that are associated with COVID-19 severity. Agent factors connected to the variation in the disease manifestations are less well recognized. The researchers until now, have no potential for the development of better, and more informative test kits. The use of bioinformatics has allowed hypotheses to be generated. More research is required to understand how these variations are important in real life. To date, this has not been thoroughly investigated. However, this article suggests that further exploration of this potential factor is vital issue. In addition, it urgently needed to categories human into six genetic groups (exploring the potential role of genome wide association, GWAS), that based on the severity of COVID-19, namely: High, moderate and low susceptible to corona virus; and low, moderate, and high resistance. The degree of severity of COVID-19 can also break down and classified based on the symptoms of COVID-19, which is include dry cough, fever, chills, cold, body ache, muscle ache, headache, loss of taste, loss of smell, tiredness, sore throat, diarrhea, nausea or vomiting, conjunctivitis, discoloration of fingers or toes, difficulty in breathing or shortness of breath, blue lips or face, excessive drowsiness, chest pain and loss of speech or movement. These symptoms vary from person to person based in their levels of immunity and genetic make-up. It was noticed that the severity of Corona virus is more in old age people with other disease conditions than in younger ones with no diseases. If the symptoms persist for more than 3 days then the person must consult a doctor immediately. People with other health conditions such as cancer, chronic kidney disease, constructive obstructive pulmonary disease (COPD), immune compromised state from solid organ transplant, obesity, heart failure, coronary artery disease, sickle cell disease, asthma, cystic fibrosis, hypertension, dementia, liver diseases, pulmonary fibrosis, pregnancy, smoking and type 1 and type 2 diabetes might be at an increased for severe illness from Covid-19. 40 Such categorization may show normal distribution curve of global human population (Figure 1). Where, the area under the curve represents $100 \%$ of the population. The $2.1 \%$ of the left area under the curve represents high susceptible persons of the population (represent 2 standard deviation below the mean, those who get sever symptoms within short period/time, and died soon after infection). Meanwhile, the right area under the curve of showed highly resistant persons that represent $2.1 \%$ of the population (represent 2 standard deviation above the mean, those who do not show any symptoms of COVID-19). A percentage of 13.6 to the left of the curve represent moderate susceptible persons (MS), (those who get moderate symptoms, and need treatment after infection). A percentage of 34.1 to the left curve represent low susceptible persons (LS), (those who showed mild symptoms then take some time to recover after treatment). A percentage of 34.1 to the right curve represent low resistant persons (LR), (those who showed mild symptoms then recovered after treatment). A percentage of 13.6 to the right represent moderate resistant individuals (MR), (those who do show mild symptoms of COVID-19 then recovered without treatment). 


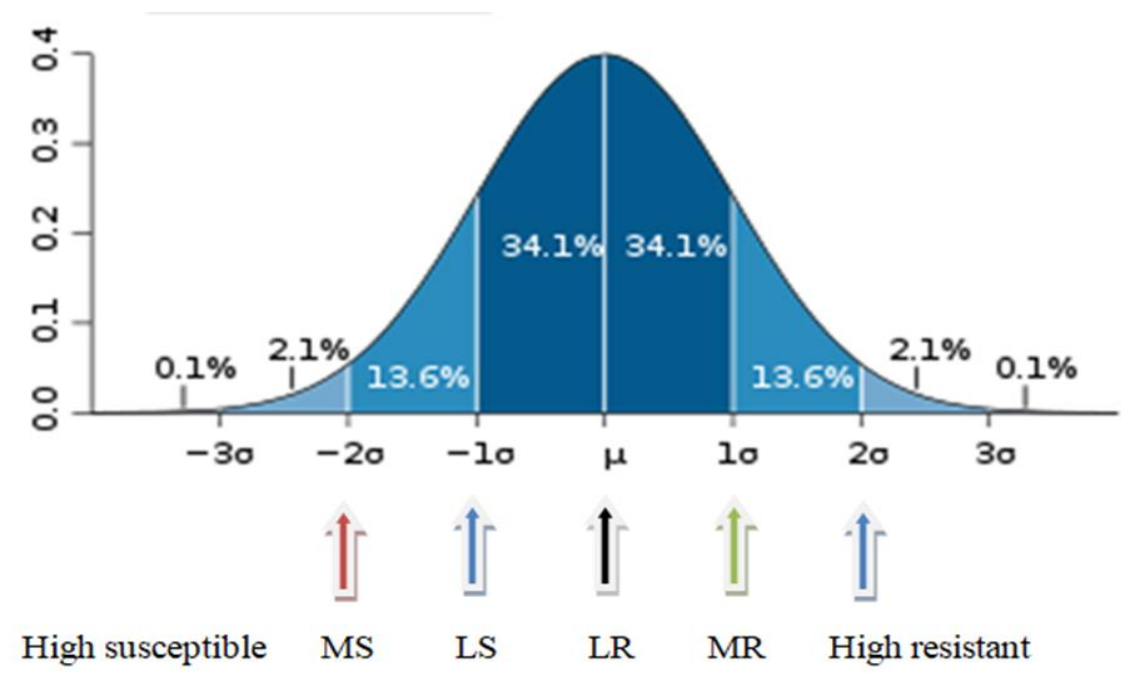

Figure 1 The area under the curve represents 100\% of the human population. The left area under the curve of high susceptible persons represents $2.1 \%$ of the population. The right area under the curve of high resistant represents $2.1 \%$ of the population. A percentage of 13.6 to the left represent moderate susceptible (MS), A percentage of 34.1 to the left represent low susceptible (LS), A percentage of 34.1 to the right represent low resistant (LR), A percentage of 13.6 to the right represent moderate resistant (MR).

According to the above genetic variations for resistant versus susceptible individuals to COVID-19 incidence, that exist between individuals and between and within communities and various regions, researchers can exploring the potential role of genome wide association, GWAS), which may play a role in answering the questions of why do the majority of people never get COVID-19? In addition, why do some people get COVID-19? The constitution of the genome through investigation GWAS for presence of susceptible gene(s) that inherited by infected individuals. So it is a vital issue to investigate the relation between resistant versus susceptible individuals to COVID-19 incidence and their molecular characterizations to identify the molecular genetic differences (genes or alleles, that are associated with resistance or sensitivity to COVID-19, as molecular genetic markers to be used as early detection of individuals susceptible to COVID19.

Furthermore, it may also suggest subdivide the above six groups into more complicated subgroups. Each group can divide into several subgroups based on suggested physiological homeostasis index. Physiological homeostasis index can be formed based on the coefficient of variation (CV) for some biological variables (body temperature, blood oxygen contents, blood pressure, blood $\mathrm{pH}$, and/or pulse rate). As any individual maintain stability (CV below 10\%) for the above biological variables, which indicate that the individual try to resists any adverse condition or outside invader to adjust his physiological condition/equilibrium that is optimal for survival. Nevertheless, one reason of homeostatic failure may be a result of high $\mathrm{CV}$ for one or more of the above biological variables. Hence, the individual may get a disease and death may ensue.

How often the biological variables should be taken. The frequency depends on the objective of the case. For routine clinical check-up, 10 samples of the variable (say blood temperature) for specific periods would give good estimate of the mean and standard deviation.

By the way CV $=\{($ standard deviation $/$ mean $) \times 100\})$.

(The value of this formula CV $=($ Standard Deviation $(\sigma) /$ Mean $(\mu)$ ) will be multiplied by 100). The coefficient of variation normalizes the standard deviation with respect to the mean. The higher the coefficient of variation, the greater the level of dispersion around the mean. Basically CV of $<10$ is very good, (indicates that the data points tend to be very close to the mean, high stability of homeostasis), 10-20 is good, 20-30 is acceptable, and CV>30 is not acceptable, (indicates that the data points are spread out over a large range of values). 


\section{Conclusion}

The result of this article revealed that the consequences of COVID-19, as an evolutionary force of natural selection may change the genetic composition of human population. It was concluded that physiological homeostasis are more stable than genetic homeostasis.

\section{Compliance with ethical standards}

\section{Acknowledgments}

The authors would like to thank Professor Dr. Abdulrazaq Abdulhameed for his guidance.

\section{Disclosure of conflict of interest}

The authors declare that they have no competing interests.

\section{References}

[1] Gordon, Betts J. Anatomy and physiology. DeSaix, Peter. Johnson, Eddie., Johnson, Jody E., Korol, Oksana., Kruse, Dean H., Poe, Brandon. Houston, Texas. 9.

[2] Martin, Elizabeth. 2008. A dictionary of biology (6th ed.). Oxford: Oxford University Press. 315-316.

[3] Biology Online. "Homeostasis". Biology Online. Retrieved 27 October 2019.

[4] Alberts, Bruce. 2002. Molecular biology of the cell (4th ed.). New York. Garland. 2002; 1292-1293.

[5] Tortora Gerard J, Anagnostakos Nicholas P. Principles of anatomy and physiology (Fifth ed.). New York: Harper \& Row, Publishers. 1987; 444-445.

[6] Jelkmann W. Erythropoietin after a century of research: younger than ever. European Journal of Haematology. 2007; 78 (3): 183-205.

[7] Armstrong CM, Cota G. Calcium block of Na+ channels and its effect on closing rate. Proceedings of the National Academy of Sciences of the United States of America. 1999; 96 (7): 4154-7.

[8] Harrison TR. Principles of Internal Medicine (third ed.). New York: McGraw-Hill Book Company. 170: 571-579.

[9] Spencer, Laci. Flotation: A Guide for Sensory Deprivation, Relaxation, \& Isolation Tanks. Lulu.com. 2015.

[10] Christopher Stephens. Natural Selection. Handbook of the Philosophy of Science. Philosophy of Biology Volume editors: Mohan Matthena.nd Christopher Stephens General editors: Dov M. Gabbai Paul Thaga,rd and John Woods. 2007.

[11] Evolution and Natural Selection. University of Michigan. 10 October 2010. Retrieved 9 November 2016. www.globalchange.umich.edu.

[12] Walton Olivia, Stevens Martin. Avian vision models and field experiments determine the survival value of peppered moth camouflage. Communications Biology. 2018; 1: 118.

[13] Orr H. Allen. Fitness and its role in evolutionary genetics. Nat Rev Genet. 2009; 10(8): 531-539.

[14] Kleinman A. The basic science and mathematics of random mutation and natural selection. Statistics in Medicine. 2014; 33 (29): 5074-5080.

[15] Eens, Marcel, PinxtenRianne. Sex-role reversal in vertebrates: behavioural and endocrinological accounts. Behavioural Processes. 2000; 51(1-3): 135-147.

[16] Barlow George W. How Do We Decide that a Species is Sex-Role Reversed?. The Quarterly Review of Biology. 2005; 80(1): 28-35.

[17] Harvey, Fiona; Carson, Mary; O'Kane, Maggie; Wasley, Andrew. MRSA superbug found in supermarket pork raises alarm over farming risks. The Guardian. 2015.

[18] Daniel LHartl,Andrew G Clark. Principles of Population Genetics. Fourth Edition. December 2006.

[19] Charlesworth B,Charlesworth D. Population genetics from 1966 to 2016. Heredity. 2017;118: 2-9. 
[20] Sarkar S. Drift and the Causes of Evolution', in P. McKay Illari, F. Russo and J. Williamson (eds.) Causality in the Sciences, Oxford: Oxford University Press. 2011; 445-469.

[21] Dong-Dong Wu, Ya-Ping Zhang. Different level of population differentiation among human genes. BMC Evolutionary Biology. 2011; 11:16.

[22] Cai JJ, Borenstein E, Chen R, Petrov DA. Similarly strong purifying selection acts on human disease genes of all evolutionary ages. Genome BiolEvol. 2009; (0): 131-144.

[23] Blekhman R, Man O, Herrmann L, Boyko AR, Indap A, Kosiol C, Bustamante CD, Teshima KM, Przeworski M. Natural selection on genes that underlie human disease susceptibility. Curr Biol. 2008; 18 (12): 883-889.

[24] Andrew Whitehead, Douglas L Crawford. Variation in tissue-specific gene expression among natural populations. Genome Biology. 6:R13. 2005.

[25] Oleksiak MF, Churchill GA, Crawford DL. Variation in gene expression within and among natural populations. Nat Genet. 2002; 32: 261-266.

[26] Oleksiak MF, Roach JL, Crawford DL. Natural variation in cardiac metabolism and gene expression in Fundulusheteroclitus. Nat Genet. 2005; 37: 67-72.

[27] Oleksiak MF, Churchill GA, Crawford DL. Variation in gene expression within and among natural populations. Nat Genet. 2002; 32: 261-266.

[28] Pritchard CC, Hsu L, Delrow J, Nelson PS: Project normal: defining normal variance in mouse gene expression. ProcNatlAcadSci USA. 2001; 98: 13266-13271.

[29] Jin W, Riley RM, Wolfinger RD, White KP, Passador-Gurgel G, Gibson G. The contributions of sex, genotype and age to transcriptional variance in Drosophila melanogaster. Nat Genet. 2001; 29: 389-395.

[30] Weiss L, (Ed). Cell and Tissue Biology: A Textbook of Histology. Baltimore, MD: Urban and Schwarzenberg. 1983.

[31] Guyton AC. Textbook of Medical Physiology. Philadelphia: W.B. Saunders Company. 1991; 8.

[32] Lee PD, Sladek R, Greenwood CMT, Hudson TJ. Control genes and variability: absence of ubiquitous reference transcripts in diverse mammalian expression studies. Genome Res. 2002; 12: 292-297.

[33] John DStorey, Jennifer Madeoy, Jeanna LStrout, MarkWurfel, James Ronald, Joshua MAkey. Gene-Expression Variation Within and Among Human. The American Society of Human Genetics. Published by Elsevier Inc. 2007.

[34] David Ellinghaus, FraukeDegenhardt, Luis Bujanda, Maria Buti, AgustínAlbillos, PietroInvernizzi, et al. Genomewide Association Study of Severe Covid-19 with Respiratory Failure. The New England Journal of Medicine. 2020.

[35] Abby Olena. Two Genetic Regions Linked with Severe COVID-19. 2020.

[36] Molly Campbell. The New England Journal of Medicineled by Professor Andre Franke, Director of the Institute of Clinical Molecular Biology (IKMB) The Scientist, E-newsletter.2020.

[37] David Ellinghaus, FraukeDegenhardt, Luis Bujanda, Maria Buti, AgustínAlbillos, PietroInvernizzi, et al. The ABO blood group locus and a chromosome 3 gene cluster associate with SARS-CoV-2 respiratory failure in an ItalianSpanish genome-wide. MedRxiv. 2020.

[38] COVID-19 Host Genetics Initiative. The COVID-19 Host Genetics Initiative, a global initiative to elucidate the role of host genetic factors in susceptibility and severity of the SARS-CoV-2 virus pandemic. European Journal of Human Genetics. 2020.

[39] Melissa L Spear, Donglei Hu, Maria Pino-Yanes, Scott Huntsman, Celeste Eng, Albert M. Levin, Victor E. Ortega, et al. A Genome-wide Association and Admixture Mapping Study of Bronchodilator Drug Response in African Americans with Asthma. Pharmacogenomics J. Pharmacogenomics J. 2019; 19(3): 249-259.

[40] Johnson Craig. Clinical Pathology of Covid19. Journal of Clinical and Experimental Pathology. $2020 ; 10$ (4): 379. 